\title{
Adolescentes que fazem uso nocivo/abusivo de álcool: percepção de risco e proteção para dependência*
}

Alcohol misuse/abuse among adolescents: risk perception and protection from addiction

Adolescentes que hacen uso nocivo/abusivo de alcohol: percepción de riesgo y protección para dependencia

Leandro Rozin ${ }^{1}$, Ivete Palmira Sanson Zagonel ${ }^{2}$

\footnotetext{
* Pesquisa realizada na Faculdades Pequeno Príncipe (FPP), Curitiba/PR, Brasil, como resultado da Dissertação do Curso de Mestrado do Programa de Pós-Graduação em Biotecnologia aplicada à Saúde da Criança e do Adolescente.

Enfermeiro, Mestre em Biotecnologia aplicada à Saúde da Criança e do Adolescente. Docente da Faculdades Pequeno Príncipe (FPP). Curitiba, PR, Brasil. E-mail: leandrorozin@bol.com.br.

${ }^{2}$ Enfermeira, Doutora em Enfermagem. Professora do Programa de Pós-Graduação em Biotecnologia aplicada à Saúde da Criança e do Adolescente da FPP. Curitiba, PR, Brasil. E-mail: ivete.zagonel@fpp.edu.br.
}

\section{RESUMO}

Objetivou compreender a percepção de adolescentes em uso nocivo e abusivo de álcool sobre os riscos a que estão expostos e proteção para dependência da substância. Estudo exploratório descritivo, quanti-qualitativo, com 280 adolescentes que responderam ao Alcohol Use Disorders Identification Test (AUDIT) e entrevista semiestruturada a 22 identificados consumidores nocivos e abusivos. Resultados indicaram adolescência conflituosa no meio familiar, associada à ausência do pai ou criação por padrastos/madrastas, desejo de autonomia e liberdade para escolhas. A experimentação do álcool prevaleceu no meio familiar, o consumo junto de amigos para momentos de diversão e comemorações, facilitados pelo acesso à compra de bebida por adolescentes. Foi identificado como proteção para consumo de álcool a atenção e aproximação dos pais e autocontrole pelos adolescentes. Recomenda-se que equipes de saúde identifiquem fatores de risco para dependência do álcool e desenvolvam ações de controle e proteção do consumo diretamente junto ao adolescente e à sua família.

Descritores: Adolescente; Risco; Transtornos Relacionados ao Uso de Substâncias; Alcoolismo; Enfermagem em Saúde Pública.

\section{ABSTRACT}

The objective was to understand the perception that adolescents with alcohol misuse and abuse behaviors have of risks and protection from addiction. This descriptive, exploratory, quanti-qualitative study was performed with 280 adolescents who answered the Alcohol Use Disorders Identification Test (AUDIT), and a semi-structured interview with 22 individuals identified as harmful and abusive consumers. Results indicated a conflicting adolescence in the family environment, associated to the father's absence or being raised by stepparents, desire for autonomy and liberty to make their own decisions. The experience with alcohol was mainly within the family environment, consuming among friends for fun, which was facilitated by the access that adolescents have to buying liquor. It was identified that the care and closeness of parents as well as the adolescents' selfcontrol protected against alcohol consumption. Health teams should identify risk factors for addiction and involve adolescents and families in control and protection actions against consumption.

Descriptors: Adolescent; Risk; Substance-Related Disorders; Alcoholism; Public Health Nursing.

\section{RESUMEN}

Se objetivó comprender la percepción de adolescentes con uso nocivo y abusivo de alcohol sobre riesgos a los que se exponen y protección para dependencia de la substancia. Estudio exploratorio, descriptivo, cuanti-cualitativo, con 280 adolescentes que respondieron al Alcohol Use Disorders Identification Test (AUDIT) y entrevista semiestructurada con 22 identificados como consumidores nocivos/abusivos. Resultados indicaron adolescencia conflictiva en ámbito familiar, asociada a ausencia del padre o crianza con padrastros/madrastras, deseo de autonomía y libertad para elecciones. El alcohol fue probado prevalentemente en ámbito familiar, consumido con amigos en momentos recreativos y festivos, facilitados por acceso a compra de bebidas por adolescentes. Fue identificado como protección para consumo de alcohol la atención y aproximación de padres y autocontrol de adolescentes. Se recomienda que equipos de salud identifiquen factores de riesgo para dependencia del alcohol y desarrollen acciones de control y protección del consumo, directamente con los adolescentes y familias.

Descriptores: Adolescente; Riesgo; Trastornos Relacionados con Sustancias; Alcoholismo; Enfermería en Salud Pública. 


\section{INTRODUÇÃO}

O álcool é a droga psicoativa mais usada na maioria dos países, tanto para a celebração como para o sofrimento, pois libera as inibições. As pessoas consomem álcool para relaxar e se divertir. Para muitos, o álcool é uma companhia nos eventos sociais e, na maior parte das vezes, o consumo de álcool implica riscos relativamente baixos, tanto para quem bebe como para terceiros. Mas, há um outro lado do uso de álcool, responsável por danos nas esferas sociais e individuais ${ }^{(1)}$.

Os prejuízos que o álcool traz para os adolescentes estão relacionados à morte por acidente, violência sexual, absenteísmo escolar, déficit de aprendizagem, além de problemas familiares, perda de emprego e prejuízo financeiro. Toda política de saúde pública que envolve o adolescente e o uso de álcool, deve ter como prioridade a mudança dos padrões de consumo, retração das quantidades consumidas e diminuição dos danos subsequentes à saúde ${ }^{(2-3)}$.

Há referência de quatro tipos de motivações do uso de álcool entre adolescentes, sendo: social, potencialização, estratégias de enfrentamento e conformidade. O motivo social inclui beber por diversão com os amigos. Motivo de potencialização envolve razões como embriagar-se e experienciar excitação, associados com uso pesado de álcool. Essas motivações estão associadas ao comportamento do adolescente(5).

Dentre os padrões de consumo de álcool que expõem o indivíduo ao risco para dependência estão o uso nocivo e o abusivo da substância. O uso nocivo de álcool foi conceituado pela Classificação Internacional de Doenças versão 10 (CID-10), como o consumo de álcool em quantidade e frequencia acima dos padrões considerados aceitáveis, causa prejuízos físicos ou mentais. Já o uso abusivo, descrito pelo Diagnostic and Statistical Manual versão IV (DSM-IV) foi definido como potencial risco para dependência e quando avaliado cuidadosamente, pode ser considerado diagnóstico de dependência, o qual caracteriza-se pelo uso contínuo, uso recorrente, perante problemas sociais, interpessoais ou físicos, associados a negligências de obrigações e até mesmo questões legais(6).

Quanto mais precoce o início do consumo de álcool, maior a probabilidade de tornar-se dependente. Com o uso constante, o organismo cria tolerância à droga, e para satisfazer (como nos efeitos iniciais) é necessário aumentar as doses, que em consequencia do uso contínuo, desenvolve a dependência pelo álcool(7).

Alguns fatores estão relacionados à dependência do álcool nesse grupo, dentre eles, a busca pela autonomia e afirmação social influenciada pelo grupo de amigos, a vivência de problemas relacionados à afetividade familiar, frustrações, baixa auto-estima, insegurança e insatisfação diante das condições de vida. Estes fatores levam os adolescentes a buscar satisfação, prazer, a demonstrar coragem e a luta para conseguir a inserção em seu grupo, o que consideram alcançar por meio do uso do álcool(8).

O uso precoce de álcool pode ser um marcador de outros fatores de risco, tornando-se indicador importante para problemas de alcoolismo na idade adulta. O consumo regular de álcool pode afetar o desenvolvimento cerebral e interromper o desenvolvimento de mecanismos comportamentais, tais como inibição dos impulsos, regulação da motivação, resultando em problemas de uso do álcool mais tarde. Alterações socioemocionais, cognitivas ou biológicas podem aumentar as chances de ocorrência de alcoolismo na idade adulta ${ }^{(9)}$.

Diante dos riscos, a abordagem do uso de álcool entre adolescentes torna-se difícil, pois o profissional de saúde restringindo suas ações nas Unidades de Saúde (US) deixa de conhecer sérios problemas de saúde que afetam aqueles que não procuram a US, principalmente os adolescentes. É preciso romper as barreiras que dividem o profissional e o adolescente, usuário do sistema público de saúde. É necessário ir até ele, conhecer a sua vida e seus projetos futuros, identificar problemas que interfiram na qualidade de vida.

A partir dessa análise da literatura, o estudo se justifica por se constatar que o consumo de álcool expõe o adolescente a inúmeros riscos multidimensionais, que reflete diretamente à responsabilidade da saúde pública. Assim, foi definido como objetivo, compreender a percepção de adolescentes em uso nocivo e abusivo de álcool sobre os riscos a que estão expostos e proteção para dependência da substância.

\section{MÉTODO}

O método utilizado para a realização do estudo foi exploratório-descritivo com análise de dados quali e quantitativos. Foi selecionado um distrito sanitário do Município de Curitiba/PR/Brasil, e aleatoriamente uma unidade de Estratégia de Saúde da Família (ESF). A partir 
dos cadastros das famílias, os pesquisadores identificaram e enviaram convite a todos os adolescentes cadastrados, com idade entre 10 e 19 anos e ambos os gêneros, para participarem do estudo, sob consentimento de seu responsável ao menor de 18 anos.

A coleta das informações, inicialmente, ocorreu por meio de um encontro realizado na escola próxima a Unidade de Saúde, que autorizou a utilização do espaço. Compareceram 280 adolescentes que aceitaram participar da primeira etapa do estudo. Essa totalidade preencheu individualmente o Alcohol Use Disorders Identification Test (AUDIT) ${ }^{(10)}$, que possibilitou identificar 28 (10\%) de adolescentes em uso nocivo e abusivo de álcool, que os expõe ao risco para dependência. O AUDIT é um teste que possibilita quantitativamente mensurar o consumo de álcool, com caráter de identificação para ações de intervenção preventivas e de controle do consumo de álcool. Sua classificação avaliativa se dá por meio de áreas de riscos para intervenções: ausência ou baixo risco (prevenção primária); uso esporádico de álcool (orientações básicas); uso nocivo de álcool (intervenção e monitoramento); uso abusivo de álcool (encaminhamento para serviço especializado).

Identificados os 28 adolescentes que faziam uso nocivo e abusivo de álcool pelo referido teste, os pesquisadores convidaram apenas esses, para segunda etapa da pesquisa: participar de uma entrevista individual e gravada, com data e horário previamente agendados, com perguntas semiestruturadas, possibilitando a liberdade para os relatos. Dos 28 adolescentes, 22 aceitaram participar da segunda etapa. As questões englobaram a percepção da vida na adolescência, os projetos futuros, a relação familiar, atividades realizadas com amigos, momento que ocorre a inserção do álcool, percepção dos efeitos e os riscos do álcool, proteção dos riscos causados pelo álcool e o que considerava necessário para aumentar a proteção quanto aos riscos.

As informações coletadas pela entrevista foram analisadas pela técnica de análise de conteúdo temática que compreende três momentos: a pré-análise, a organização do material existente para análise que pode ser flexível; a exploração do material, identificação da comunicação, momento em que surge a categorização; e o tratamento dos resultados, a inferência e a interpretação que resulta em inferências e interpretação de todos os depoimentos(11).

Foram respeitados os princípios éticos, seguindo a normas de pesquisa em saúde referidas pela Resolução $n^{\circ}$ 196 do Conselho Nacional de Saúde. O projeto foi encaminhado e aprovado pelo Comitê de Ética em Pesquisa do Hospital Pequeno Príncipe com o parecer $n^{\circ}$ 0811-2010. A coleta das informações foi realizada após consentimento do adolescente e/ou do responsável (quando menor de 18 anos). Estes foram entrevistados separadamente dos pais, раra que não houvesse intimidação diante dos pais e ocorresse omissão de informações e para que os resultados da pesquisa pudessem alcançar os objetivos propostos.

Para apresentar os discursos, mantendo a privacidade e o anonimato dos participantes, foi utilizada iniciais "Ad" referente à adolescente, sequenciado por números de um a 22 aleatoriamente conforme as participações no estudo.

\section{RESULTADOS}

A aplicação do questionário (AUDIT) constatou que $10 \%$ dos adolescentes faziam uso nocivo e abusivo de álcool, expresso na Tabela 1.

Tabela 1: Distribuição da amostra conforme consumo de álcool. Curitiba, PR, 2011.

\begin{tabular}{ccc}
\hline Teste de identificação sobre o uso de álcool & $\mathbf{N}$ & $\mathbf{\%}$ \\
\hline Ausência ou uso de álcool & 200 & 71,43 \\
Consumo esporádico de álcool & 52 & 18,57 \\
Consumo nocivo de álcool & 21 & 7,50 \\
Consumo abusivo de álcool & 07 & 2,50 \\
\hline Total & $\mathbf{2 8 0}$ & $\mathbf{1 0 0}$ \\
\hline
\end{tabular}

A prevalência etária dos 28 adolescentes consumidores nocivos e abusivos de álcool foi identificada acima de 15 anos correspondendo a $71,43 \%$ $(n=20)$. No gênero feminino $60,71 \%(n=17)$, comparado com 39,29\%( $n=11)$ masculino faziam uso nocivo e abusivo da substância. Dos adolescentes que faziam uso nocivo, o gênero feminino foi predominante com $76,20 \%$ ( $n=16)$, já em uso abusivo, observou-se que o masculino teve maior predominância $71,43 \% \quad(n=5)$. Quanto ao período de estudo, houve prevalência de $46 \% \quad(n=13)$ entre adolescentes que estudavam no período noturno. 
A análise qualitativa resultou em uma operação de classificação de elementos constitutivos de um conjunto de depoimentos, por diferenciação e, seguidamente, por reagrupamento segundo analogia dos achados. Foi possível apontar três temas emergidos das Unidades de
Significação: percepção de vida pelos adolescentes em risco para dependência de álcool; percepção do adolescente sobre os riscos a que está exposto para dependência e, percepção dos fatores de proteção quanto aos riscos de dependência do álcool (Figura 1).

Figura 1: Percepção dos adolescentes quanto aos riscos para e da dependência do álcool e fatores de proteção. Curitiba, PR, 2011.

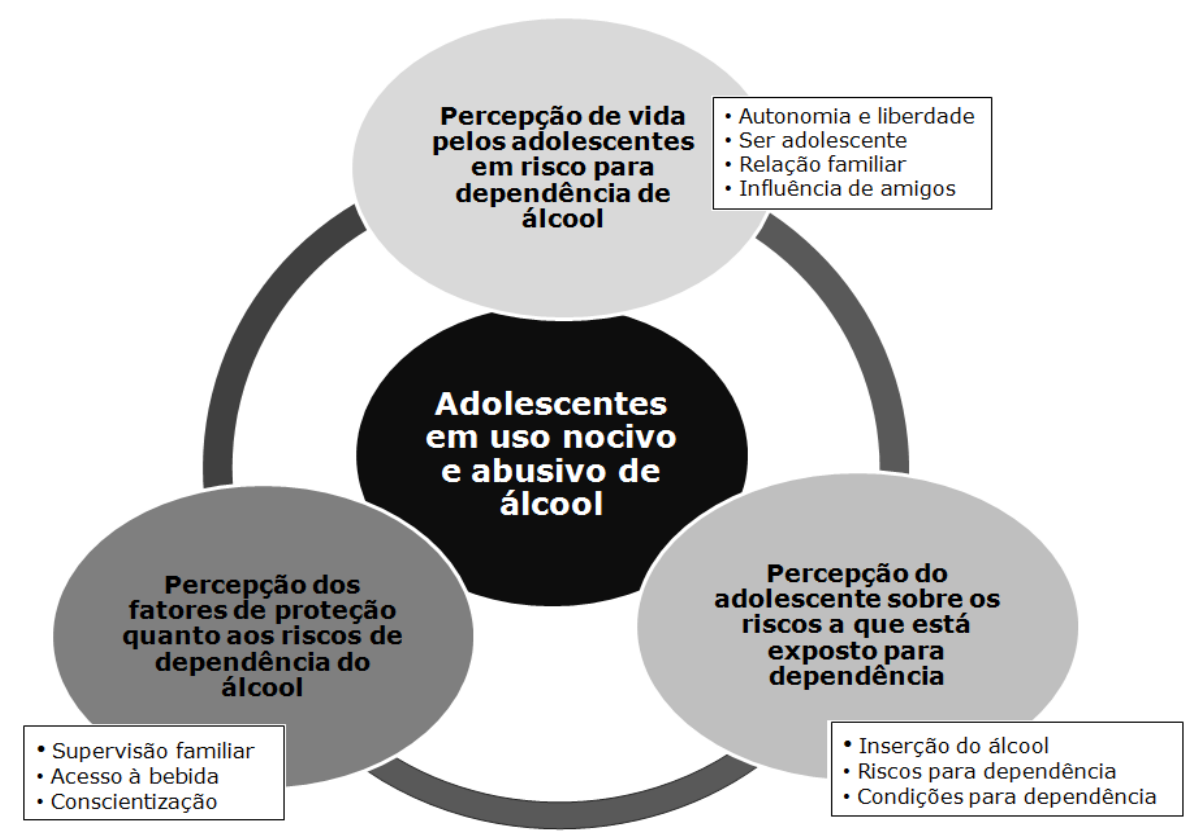

\section{Percepção de vida pelos adolescentes em risco para dependência de álcool}

Os adolescentes em risco para dependência de álcool relacionam a fase da adolescência com o surgimento de responsabilidades, associada ao controle ainda presente dos pais. Relatam que na adolescência, ora são tratados como criança, ora como adultos. Também, referem pensar no futuro, como serão quando adultos, projeções atreladas à autonomia, responsabilidade e exigência de mais liberdade.

Ah, eu gosto, posso fazer mais coisas sem ser tão regulado pelos meus pais, só que ainda tem que pedir tudo, e eles me tratam como criança ainda [...] (Ad1).

[...] com o tempo temos certas responsabilidades. Na adolescência devemos pensar o que queremos no futuro, ter mais responsabilidades [...] (Ad3).

Também foram constatados, na vivência do adolescente em risco para dependência ou dependência de álcool, conflitos relacionados à baixa autoestima, desesperança e solidão (sensação de abandono).
A minha vida é meio conturbada, cheia de conflitos, com altos e baixos [...] não sei, as vezes da uma tristeza, só pensando em coisas ruins. (Ad2).

[...] ninguém dá a mínima pra mim [...](Ad6).

Em relação aos projetos futuros de adolescentes em risco para dependência de álcool, apreende-se a positividade de objetivos, idealização de "sonhos" para a idade adulta, relacionados a vida profissional qualificada, trabalho, vida financeira e material estáveis. A liberdade é consideravelmente almejada como planos futuros dos adolescentes. Além disso, alguns associam a felicidade com a formação futura de novas famílias.

Terminar a escola, fazer faculdade e trabalhar [...] Ah, também quero casar e ter filhos, sei lá, ser feliz! (Ad1).

[...] ter uma casa, carro, trabalhar e ganhar dinheiro (Ad7).

[...] quero ter um carro, poder sair de noite e me divertir mais, com mais liberdade (Ad14).

A relação familiar referida pelos adolescentes é permeada de conflitos intrafamiliares. O fator mais 
significativo identificado entre os depoimentos é a ausência da figura paterna no contexto familiar.

[...] os meus irmãos, são insuportáveis, se eu pudesse já tinha matado minha irmã junto com a minha mãe [...]. Meu pai não mora comigo. (Ad8).

[...] brigo muito com minha mãe e meu irmão [...] Não sei se é pela falta do meu pai [...] (Ad11).

Os relacionamentos familiares conturbados referemse também, aos conflitos de convivência e criação por padrastos e madrastas. Os depoimentos denotam controle, estresse, dificuldades relacionais, maus tratos.

[...] A relação com minha madrasta é um saco, ele [pai] só quer me controlar e que eu trabalhe [...] (Ad6).

[...] meu padrasto, sempre foi um péssimo pai [...]sempre me maltratou [...] (Ad13).

Além de conflitos familiares, a influência de amigos e/ou colegas na adolescência foi um aspecto influenciador para o uso e abuso de álcool. É possível identificar pelos depoimentos que um número considerável de adolescentes em risco para dependência de álcool, relatam a associação entre beber em bares, festas, com a presença de amigos.

[...] vou em festas e a gente costuma se reunir na casa de alguém pra beber e vamos pra praça também nos fins de semana [...](Ad8).

A gente vai na praça, as vezes em algum parque, ficar na panificadora, barzinho e festas, bebendo (Ad22).

\section{Percepção do adolescente sobre os riscos a que está exposto para dependência}

Os adolescentes ao serem questionados sobre os momentos de contato e inserção do álcool em suas vidas, relataram que ocorreu no ambiente familiar em situações cotidianas e em companhia de amigos, como forma de diversão e lazer. É possível apreender que o consumo se estende também aos momentos em que está sozinho, mantendo o hábito mesmo longe dos amigos.

Em casa mesmo, quando tem churrasco, tem cerveja, vinho que meus pais tomam as vezes. [...] Bebo sozinha ou com meus amigos (Ad6).
[...] Nos aniversários ou quando ficamos sozinhos em casa ou na casa do meu amigo a gente toma escondido também (Ad14).

Mais em festas, e a noite quando a gente se encontra também. Mas eu bebo também em casa, nos finais de semana, com meu pai e meu irmão (Ad17).

A percepção do risco para dependência ou dependência de álcool entre adolescentes, é referida como a ocorrência de problemas consequentes ao consumo, a imagem negativa da dependência da substância, além de seus malefícios. Há consciência dos riscos, porém estes são referidos como acontecendo no outro e não em si mesmo. Conhecem os malefícios, como descontrole, problemas para a família, prejuízos ao organismo, agressividade, destruição dos projetos futuros, perda de limites, inconsequência dos atos, domínio dos amigos.

Os jovens estão bebendo cada vez mais cedo, e de forma inconsequentes e descontrolada. [...] os abusos acontecem a partir da liberdade, saindo para festas com amigos, etc. [...] (Ad2).

Sei que o alcoolismo traz problemas para a família do dependente e pra ele mesmo, traz dependência e afeta o organismo [...] (Ad4).

Os relatos demonstram que a dependência de álcool, está relacionada ao beber precocemente, a facilidade do acesso ao álcool e no beber constantemente. Além disso, os adolescentes em risco para dependência do álcool descrevem suas percepções sobre as condições para tornar-se dependentes. Referem condições genéticas, expectativa de melhorar as situações difíceis, problemas relacionais familiares, falta de controle.

[...] a dependência é hereditária, eu tive casos de alcoólatras na família [...] (Ad2).

Tem relação com algum problema que essa pessoa tem, e só fica melhor quando bebe, e isso fica um ciclo vicioso. Problema em casa, coisas pessoais mal resolvidas [...] (Ad15).

[...]Quem bebe todo dia e não consegue controlar [...] (Ad20). 


\section{Percepção dos fatores de proteção quanto aos riscos de dependência do álcool}

Como fator de proteção, os adolescentes revelam não fazer uso diário ou não usar de forma pesada constantemente, além de não ser influenciado por amigos. Na concepção dos adolescentes, também deixam claro que beber concomitantemente ou socialmente não os expõe a riscos de dependência do álcool.

[...] Não ir na onda dos amigos, não beber só porque os amigos bebem, você beber também para se tornar popular! (Ad3).

Não consumindo em excesso, só tomando socialmente (Ad9).

Porém, quando o questionamento é direcionado para si, é constatada a dificuldade no controle do uso de álcool.

[...] quando eu bebo, tento manerar e não exagerar na dose [...] mas na maioria das vezes uns gole chama outros e quando vê, já era! (Ad15).

Alguns desses adolescentes descrevem não se proteger рага os riscos de dependência do álcool a que estão expostos. Observa-se em algumas falas a desmotivação; em outras, beber é comum socialmente.

Não me protejo, quando me da vontade de beber eu bebo. [...] todo mundo faz isso! (Ad5).

[...] Acho que é uma fase mesmo, todos meus amigos usam e duvido quem não bebe na minha idade [...](Ad17).

Com a identificação das necessidades para aumentar a proteção desses adolescentes quanto aos riscos da dependência do álcool são apontadas medidas familiares na ótica dos adolescentes que fazem uso nocivo e abusivo de álcool. Dentre elas, são destacadas a importância da atenção, orientações e supervisão e acompanhamento dos pais na vida dos adolescentes.

[...]os pais devem proibir e fazer os adolescentes pensar no que estão fazendo com suas vidas para depois não se arrependerem (Ad3).

[...] os pais tinham que participar mais da vida dos filhos $e$ saber com quem eles andam, o que usam ou o que fazem, $e$ onde vão (Ad16).
Também foi relatado, como fator de proteção para dependência de álcool, a necessidade do controle na venda das bebidas para menores de 18 anos, conforme a legislação vigente. As falas dos adolescentes denunciam a facilidade no acesso a bebida e sugerem mais fiscalização em locais que vendem bebidas.

Mais fiscalização nos bares, para não vender para menores, porque apesar de ter várias leis, elas não são respeitadas. [...](Ad5).

[...]Ninguém pergunta minha idade quando vou comprar e eu sei que não pode (Ad19).

A necessidade de conscientização dos malefícios que o álcool causa, são relatadas pelos adolescentes para proteção contra a dependência de álcool. Nas falas observamos a necessidade da autoconsciência atrelada ao conhecimento dos riscos e prejuízos causados pela bebida.

[...]Ter palestras que mostrem os riscos que o álcool pode trazer para vida (Ad4).

Não ser influenciado pelos amigos, ser consciente do que faz, ter em mente suas consequências[...]. (Ad7).

\section{DISCUSSÃO}

A adolescência é uma nova estação do ser, a preparação para idade adulta, com aumento de autonomia e responsabilidades nas vivências familiares, na escola e no meio social, tendo em mãos o potencial para vida adulta. É fundamental para adolescentes pertencer a um grupo, isso frequentemente é o ponto de partida para conflitos familiares e atinge o seu pico no meio da adolescência. Nesse grupo social, os adolescentes encontram similaridade em características de pensar e agir, tentando encontrar espaço e sua identidade $^{(12)}$.

Importante salientar, que a adolescência é uma das etapas do ciclo evolutivo em que o ser humano vivencia a saída de casa, para conviver com os amigos, com a turma, porém os vínculos com os pais permanecem e são sentidos quando começam e perder a dependência rumo à independência adulta. Diante dessas mudanças pensam no futuro, incluindo estudos, trabalho e ganho financeiro. Esse processo é percebido como de mudanças significativas no modo de pensar, agir, exigindo maiores responsabilidades, perspectivas futuras e a consciência 
do certo e errado. Os adolescentes vêem o futuro associado à educação como elemento central, indicando que o estudo é visto como um facilitador da ascensão social, garantia por melhor emprego ou profissão, associada à busca de sucesso financeiro(13).

O fator da ausência do pai no contexto familiar está diretamente ligado entre adolescentes que fazem uso e abuso de álcool. Fatores de risco para consumo e uso abusivo de álcool entre adolescentes estão relacionados à famílias disfuncionais, como laços familiares conflitivos e com pouca proximidade entre os membros, frieza afetiva dos pais, rejeição, conflitos na estrutura familiar relacionada a busca pela autonomia dos adolescentes com ausência ou negligência de controle ou monitoramento dos pais. Além disso, a falta de uma hierarquia bem definida e pais que não dão exemplo positivo quanto ao uso de substâncias ${ }^{(14-16)}$.

Como fatores de proteção, referem as demonstrações de afeto com os filhos, dar atenção sobre o que os filhos gostam e abertura na comunicação e existência de normas. $O$ modelo dos pais e comportamentos relacionados à afetividade, comunicação familiar e práticas disciplinares, predizem importantes mediadores na formação do adolescente. Assim, adolescentes criados por pais biológicos têm menos risco para uso de substâncias em relação a adolescentes educados por padrastos e madrastras. Além disso, a criação por pais não biológicos que fazem uso de álcool, influenciam para uso e abuso da substância entre os adolescentes ${ }^{(17-18)}$.

Após a puberdade, o indivíduo muda aos poucos a sua maneira de ser, em movimentos que iniciam do seu interior e por fim, acabam se exteriorizando, fazendo com que o adolescente busque um meio, ao qual se identifique. Amigos e colegas são fortes influências para a maioria dos adolescentes e jovens que iniciam e usam constantemente o álcool, motivados pela busca da diversão, prazer, quebra de rotina e redução da ansiedade e estresse $^{(12)}$.

Entre as influências para o abuso de álcool, indicam a associação entre companhias de uso de álcool e o fácil acesso em bares e festas que dispõem de álcool. Estes são fatores negativos e de forte influência para uso da substância entre adolescentes e jovens. Adolescentes são motivados para o uso de álcool pela curiosidade, e com maior frequência para ficar animados, sentir prazer e diminuir ansiedade ${ }^{(14-15,19-20)}$.
Os jovens não desenvolvem problemas simplesmente por motivo natural, mas sim pelas referências sociais, que influenciam na identidade e na vida dos adolescentes. 0 maior contato dos jovens ocorre com a influência de amigos e de familiares. Nesse sentido, o uso de álcool tem influência do contexto vivido entre aspectos familiares e sociais, principalmente entre o grupo de amigos ${ }^{(21)}$.

Em nosso estudo, por se tratar de adolescentes em risco para dependência de álcool, observou-se a visão negativista sobre a dependência da substância. Referiram-se a avaliação no outro ou no grupo (dos adolescentes), e não sobre a percepção de si em relação ao consumo nocivo e abusivo do álcool ou dos riscos a que estava exposto.

Em estudo similar foi descrito que a dependência do álcool no outro, foi vista pelos adolescentes como um estigma, imoral e de natureza psicossocial, com predomínio de imagem negativa ${ }^{(22)}$. Porém, a autopercepção de uso concomitante do álcool não é vista pelos adolescentes como uma droga potencial de riscos à saúde, além de muitos não classificarem o álcool como droga, demonstram ser invulneráveis e onipotentes em relação a substância(23).

Além do estímulo dos meios de comunicação e da condescendência dos pais, é possível identificar outros fatores de risco que viabilizam o acesso dos adolescentes ao álcool, como sua disponibilidade por ser uma droga lícita de baixo custo, vendida em estabelecimentos comerciais, falta de fiscalização adequada para sua venda, sendo comum a compra por menores de 18 anos(22-24).

A dependência do álcool não ocorre por fator único, mas sim por uma rede multifatorial. A adolescência é considerada um fator de risco para o consumo de álcool, associado às condutas desenvolvidas nessa fase de transição para a idade adulta. Entre os fatores influenciadores no consumo e na dependência do álcool, têm-se os sociais, ambientais e genéticos.

\section{CONCLUSÃO}

Adolescentes que fazem uso nocivo e abusivo de álcool indicaram a adolescência como uma fase conflituosa no meio familiar relacionada à ausência de figura paterna e criação por padrastos ou madrastas, com desejo de autonomia e liberdade para escolhas e perspectivas positivas em relação ao futuro, idealizadas com a vida profissional qualificada, trabalho, vida financeira e material estáveis. Demonstraram que o 
contato com o álcool ocorre no meio familiar, em finais de semana ou momentos de comemoração, mas também é evidenciado quando em companhia de amigos, associado aos encontros para diversão.

Como fatores de proteção para dependência do álcool constatou-se a atenção e o controle familiar; não ser influenciado por amigos; fiscalização e controle na comercialização de bebidas alcoólicas e conscientização sobre o consumo de álcool. A dependência do álcool é vista como negativa no outro, porém não tem autopercepção do risco a que estão expostos, pois fazem uso nocivo e abusivo da substância.

O conhecimento dos riscos em que os adolescentes estão expostos e de proteção para consumo de álcool torna possível o planejamento de ações eficazes na prevenção e controle do consumo que pode acarretar na dependência de álcool, enquanto adolescentes ou quando adultos. $O$ estudo retrata a identificação do uso nocivo e abusivo da substância e investiga os riscos em que adolescentes estão expostos, à voz de adolescentes

\section{REFERÊNCIAS}

1. Stronach B. Álcool e redução de danos. In: Buning $M$, et al. Álcool e redução de danos: uma abordagem inovadora para países em transição. Secretaria de Atenção à Saúde.

Departamento de Ações Programáticas Estratégicas. Brasília: Ministério da Saúde, 2004, p.29-35.

2. Pechansky F, Szobot CM, Scivoletto S. Uso de álcool entre adolescentes: conceitos, características epidemiológicas e fatores etiopatogênicos. Rev. Bras. Psiquiatr. 2004; 26 (Supl I), 14-17.

3. Reimuller A, Hussong A, Ennett ST. The influence of alcoholspecific communication on adolescent alcohol use and alcoholrelated consequences. Prev Sci. 2011;12(4):389-400.

4. Osiatynska E. Álcool e saúde. In: Buning, M. et al. Álcool e redução de danos: uma abordagem inovadora para países em transição. Secretaria de Atenção à Saúde: Departamento de Ações Programáticas Estratégicas. Brasília: Ministério da Saúde, 2004. p.37-47.

5. Patrick ME, Schulenberg JE, O'Malley PM, Johnston LD, Bachman JG. Adolescents' reported reasons for alcohol and marijuana use as predictors of substance use and problems in adulthood. J. Stud. Alcohol Drugs. 2011; 72:106-116.

6. Babor TF, Higgins-Biddles JC, Saunders JB, Monteiro MG. AUDIT: Teste para Identificação de Problemas Relacionados ao Uso de Álcool - Roteiro para Uso em Atenção Primária. Ribeirão Preto: PAI-PAD; 2003.

7. Lacerda RB. Efeitos de substâncias psicoativas no organismo. SUPERA: Sistema de detecção do Uso abusivo e dependência de substâncias Psicoativas: Encaminhamento, intervenção breve, Reinserção social e Acompanhamento. Brasília: Ministério da Saúde, 2008. 78p.

8. Saito MI, Silva LEV, Leal MM. Adolescência: prevenção e risco. $2^{\text {th }}$.ed. São Paulo: Atheneu; 2008. 601p.

9. Guttmannova K, Bailey JA, Hill KG, Lee JO, Hawkins JD, Woods ML, et al. Sensitive periods for adolescent alcohol use initiation: predicting the lifetime occurrence and chronicity of que vivenciam essa vulnerabilidade. Contribui como indicador as equipes de saúde para atuar com maior atenção e dinamismo na saúde do adolescente.

No Brasil, as ações públicas de saúde têm foco descentralizado como forma de estar mais perto da população e intervir nas problemáticas identificadas. Cabe aos serviços e profissionais de saúde, com maior proximidade da população, intervir com educação em saúde e acompanhamento dos adolescentes expostos aos riscos, bem como de suas famílias e atuar no controle do uso de álcool.

Ações preventivas e de controle sobre o consumo de álcool tornam-se possíveis quando há efetivamente profissionais capacitados que assistam individualmente e/ou em grupos essa faixa etária. Novos estudos devem ser conduzidos no sentido de contemplar ações preventivas e de controle do uso de álcool em adolescentes nas redes básicas dos serviços de saúde envolvendo equipe de saúde com preparo específico para este tipo de agravo.

alcohol problems in adulthood. Stud. Alcohol Drugs. 2011; 72: 221-231.

10. Ronzani TM, Paiva FS, Cotta JMO, Bastos RR. Expectativas sobre os efeitos do uso de álcool entre adolescentes. Psicologia em Pesquisa. 2009; 3(01): 75-86.

11. Bardin L. Análise de conteúdo. Lisboa (Portugal): Edições 70; 2010.

12. Rozin L, Zagonel IPS. Fatores de risco para dependência de álcool em adolescentes. Acta Paul Enferm. 2012; 25(2):314-8.

13. Pratta EMM, Santos MA. Opiniões dos adolescentes do ensino médio sobre o relacionamento familiar e seus planos para o futuro. Paidéia. 2007; 17(36): 103-114.

14. Ruiz MR, Andrade D. La familia y los factores de riesgo relacionados con el consumo de alcohol y tabaco en los niños y adolescentes (Guayaquil-Ecuador). Rev. Latino-Am.

Enfermagem. 2005; 13(número especial): 813-8.

15. Fraga S, Sousa S, Ramos E, Dias S, Barros H. Alcohol use among 13-year-old adolescents: associated factors and perceptions. Public Health. 2011;125:448-456.

16. Skinner E, Johnson S, Snyder T. Six dimensions of parenting: a motivational model. Parent Sci Pract. 2005; 5(2):175-235.

17. Arias NM, Ferriani MGC. Factores protectores de las familias para prevenir el consumo de drogas en un municipio de Colombia. Rev. Latino-am. Enfermagem. 2010; 18(Spec):504-12. 18. Chiapetti N, Serbena CA. Uso de álcool, tabaco e drogas por estudantes da área de saúde de uma universidade de Curitiba. Psicol. Reflex. Crit. 2007;20(2):303-313.

19. Matos A M, Carvalho RC, Costa MCO, Gomes KEPS, Santos LM. Consumo frequente de bebidas alcoólicas por adolescentes escolares: estudo de fatores associados. Rev Bras Epidemiol. 2010;13(2):1-12.

20. Chartier KG, Hesselbrock MN, Hesselbrock VM. Development and vulnerability factors in adolescent alcohol use. Child Adolesc Psychiatr Clin N Am. 2010;19(3): 493-504. 21. Aguiar WMJ, Bock AMB, Ozella S. Orientação profissional com adolescentes: um exemplo de prática na abordagem sócio- 
histórica. In: Bock AMB, Gonçalvez MGM, Furtado O. Psicologia sócio-histórica: uma perspectiva crítica em psicologia. São Paulo: Cortez;2001. p.163-178.

22. Peluso ETP, Blay SL. Public perception of alcohol dependence. Ver. Bras. Psiquiatr. 2008;30(1):19-24.

23. Casswell S, You RQ, Huckle T. Alcohol's harm to others: reduced wellbeing and health status for those with heavy drinkers in their lives. Addiction. 2011;106(6): 1087-94.

24. Alavarse GMA, Carvalho MDB. Álcool e adolescência: o perfil de consumidores de um município do norte do Paraná. Esc. Anna Nery. 2006;10(3):408-16.

Artigo recebido em 14/08/2012.

Aprovado para publicação em 28/05/2013.

Artigo publicado em 30/09/2013. 